\title{
INFLUENCIA DE FACTORES INSTITUCIONALES Y DE CONVENIENCIA EN LA ACEPTACION DE PLANIFICACION FAMILIAR EN EL POST-PARTO
}

\author{
Dr. Enrique Martínez Velásquez - M.D., M.S.P.* \\ Dr. Anthony Measham - M.D., Dr. P.H.**
}

Un informe anterior (1) examinaba las relaciones entre ciertas características socio-demográficas de la mujer y la aceptación de Planificación Familiar en tres hospitales de maternidad en Medellín - Colombia, una ciudad con un poco más de un miIlón de habitantes. Este texto presenta datos adicionales derivados del estudio, relacionados con dos temas generales: Conocimiento de la' Institución y sus Servicios de Planificación Familiar, y Percepción de la Conveniencia de la Clínica de Post-Parto.

\section{Diseño de la investigación y metodología}

En cada institución se seleccionaron 100 nombres continuos de adelante para atrás, tanto para aceptantes como para no aceptantes tomados del registro diario de trabajo. (Esto en la Clínica Luz Castro y en el Hospital San Vicente). En el Instituto Colombiano de Seguros Sociales, como el programa se había terminado para el momento de la encuesta, ya no se tenían registros diarios de trabajo. Se hizo un listado de 200 nombres de partos o abortos ocurridos de la fecha hacia atrás, según los registros del ICSS.

\section{CLINICA LUZ CASTRO:}

No aceptantes; se escogieron 100 nombres, se visitaron 80 señoras para obtener 75 encuestas. Aceptantes; se escogieron 100 nombres, se visitaron 79 para obtener 75 encuestas.

\section{HOSPITAL SAN VICENTE:}

No aceptantes; se escogieron 100 nombres, se visitaron 86 para obtener 75 encuestas. Aceptantes; se escogieron 100 nombres, se visitaron 91 para obtener 75 encuestas.

INSTITUTO COLOMBIANO DE SEGUROS SOCIALES :

Se visitaron de los 200 nombres hasta obtener 75 aceptantes y 28 no aceptantes, como las no aceptantes manifestaban que aún no habían ido al programa y serían aceptantes, se decidió poner una encuestadora en el programa para que tomara allí mismo la encuesta de las señoras que no fueran aceptantes hasta completar las 75. De las visitas hechas, 49 no tenían dirección exacta y no pudieron localizarse.

\footnotetext{
* Profesor Escuela Nacional de Salud Pública, Medellín - Colombia.

* Asesor Residente del Consejo de Población, Bogotá - Colombia.
} 
En total se hicieron 488 visitas a hogares para obtener 403 entrevistas efectivas; las 47 entrevistas faltantes se hicieron directamente en la consulta del Instituto Colombiano de Seguros Sociales a no aceptantes. El $76 \%$ de las mujeres fueron entrevistadas entre el primero y el cuarto mes de post-parto o del post-aborto por seis (6) estudiantes universitarias de comunicaciones.

\section{Resultados:}

El estudio señaló la dificultad en definir con fines operacionales, una no-aceptante de Planificación Familiar (2). Con el fin de facilitar la comparación de aceptantes y no aceptantes proveeremos primeramente datos ilustrativos de lo que la "no aceptación" significa en el contexto de este estudio. (Aceptantes fueron aquellas mujeres que adoptaron un método de planificación familiar en el Programa Post-Parto). La Tabla No 1 muestra las respuestas a la pregunta: ¿Piensa Usted practicar la Planificación Familiar en un futuro? La cual fue formulada a todas las 225 no aceptantes.

\section{TABLA Nㅇ 1}

PLANES DECLARADOS POR LAS NO ACEPTAN. TES RESPECTO A LA PRACTICA FUTURA DE PLANIFICACION FAMILIAR

\begin{tabular}{lcr}
\hline Planes Futuros & Porcentaje & $\mathbf{n}$ \\
\hline No piensa practicar & 13 & 29 \\
No sabe & 46 & 103 \\
Piensa practicar: & 16 & 35 \\
De 1 a 3 meses & 4 & 8 \\
De 4 a 12 meses & 8 & 19 \\
Después de 1 año & 8 & 19 \\
Después de amamantar & 5 & 12 \\
Aciualmente practica & 100 & 225 \\
TOTAL & & \\
\hline
\end{tabular}

Puede verse que el $5 \%$ de las mujeres estaban actualmente practican- do (la mayoría adoptaron la planificación familiar por fuera del Programa Post-parto), que el $35 \%$ planeaban practicarla en el futuro y el $46 \%$ "no sabían". Así pues, únicamente el $13 \%$ de las mujeres, aquellas que dijeron no tener intención de practicarla, pueden ser categorizadas como claramente negativas hacia la planificación familiar. La Tabla № 2. suministra las razones aludidas para no adoptar la planificación familiar con anterioridad a la entrevista.

TABLA N $: 2$

RAZON PRINCIPAL DECLARADA PARA IESPERAR - para no planear El uso de la ANTICONCEPCION

\begin{tabular}{lcc}
\hline Razón & Porcentaje & n \\
\hline Separada o viviendo sola & 22 & 42 \\
La familia se opone o no & & \\
se ha discutido aún el tema & 19 & 38 \\
Otros (v.gr. está en dieta) & 19 & 38 \\
Desea más hijos & 15 & 29 \\
Está amamantando & 14 & 28 \\
Los métodos son nocivos & 5 & 10 \\
Esperando una cita de la & 3 & 5 \\
clínica & 3 & 5 \\
Está embarazada & 100 & 195 \\
\multicolumn{2}{l}{ TOTAL } & \\
\hline
\end{tabular}

Más de dos tercios del mayor grupo de la Tabla 2, aquellas separadas o viviendo solas, dijeron no conocer sus planes futuros respecto a la planificación familiar. Casi la misma proporción del siguiente grupo por tamaño (oposición de los padres, etc.) produjeron la misma respuesta. Una proporción similar de aquellas que deseaban más hijos, o no planeaban practicar la planificación familiar o no estaban seguras. Estos grupos parecen ser menos positivos hacia la planificación familiar que el de aquellas que estaban amamantando, de las cuales más de los dos tercios dijeron tener intención de practicarla. Unicamente una de las $10 \mathrm{mu}$ - 
jeres que consideraban nocivos los métodos, en comparación con todas las 5 que estaban esperando una cita, planeaba practicar. Finalmente, únicamente una de las 3 mujeres embarazadas esperaba adoptar la planificación familiar en el futuro. Los hallazgos importantes aquí parecerían ser la creencia de las mujeres viviendo solas, de que no necesitaban protección y una aparente demostración adicional de que la oposición familiar (o la falta de aprobación) es un impedimento que opera en contra de la aceptación.

\section{FACTORES INSTITUCIONALES $Y$ LA ACEPTACION DE PLANIFICACION FAMILIAR}

\section{Conocimiento del Programa Post- Parto de Planificación Familiar}

El noventa y uno (91\%) por ciento de las no aceptantes sabían que había una clínica de planificación familiar en el hospital donde fueron atendidas. Sinembargo, mientras que el $59 \%$ de las aceptantes habían oído de los Servicios de planificación familiar con más de un año de anterioridad a la entrevista, únicamente el $35 \%$ de las no aceptantes habían sabido acerca de su disponibilidad durante el mismo lapso de tiempo. Igualmente, mientras que el $63 \%$ de las aceptantes estaban enteradas de los servicios antes de su último embarazo (para el cual fueron hospitalizadas), tal fué el caso con únicamente el $42 \%$ de las no aceptantes. Ambos hallazgos tienen significancia estadística (para la prueba de $X^{2} p<$ .001 en cada caso). La probabilidad de haberse enterado de los servicios por intermedio de amigos y parientes fue mayor para las aceptantes que para las no aceptantes, siendo los porcentajes 42 y 33 respectivamente, diferencia que tiene igualmente significancia estadística (para $X^{2} p<.05$ ).
(La mayoría se enteraron por medio de personal del hospital - 44 y 48 por ciento respectivamente).

Lo anterior está de acuerdo con la frecuente conclusión de que amigos y parientes ejercen una influencia considerable sobre la decisión de adoptar la planificación familiar (3). Esta noción recibió apoyo adicional del hecho de que el $51 \%$ de las aceptantes vs. $29 \%$ de las no aceptantes tenían amigos o parientes quienes habían utilizado los servicios de planificación familiar (la diferencia tiene significancia estadística: para $X^{2}, p<$ $001)$. Entre éstos, una mayor proporción $(33 \%)$ de amigos y parientes de las no aceptantes habían encontrado dificultades con el método de planificación familiar que de amigos y parientes de las aceptantes (25\%).

Los hallazgos relacionados con el momento en que la mujer supo por primera vez acerca de los servicios de planificación familiar apoyan la noción de que el conocimiento de disponibilidad está positivamente asociado con la aceptación, y quizás también de que la decisión de practicar la planificación familiar es tomada después de un período latente de meses o años.

\section{Información y Educación en la Sala de Maternidad}

\section{a) Reacción a Varios Medios:}

Un poco más de aceptantes que de no aceptantes ( 66 vs. 61 por ciento) tuvieron una charla personal sobre planificación familiar con personal del hospital. Entre éstas, las aceptantes mostraron tendencia a tener conversaciones más largas: $56 \%$ de las aceptantes vs. $40 \%$ de las no aceptantes conversaron por más de $10 \mathrm{mi}$ nutos (una diferencia con significancia estadística: para $\left.X^{2}, p<.01\right)$; 33 y $32 \%$, respectivamente, de 5 a 10 
minutos; 11 y $21 \%$ respectivamente, menos de 5 minutos. Una explicación lógica para esta diferencia es que las aceptantes mostraron evidentemente un mayor interés en la charla, presumiblemente como resultado de una mayor motivación hacia la planificación familiar. Más aceptantes ( $45 \%$ ) que no aceptantes (31\%) afirmaron que había habido una charla de grupo sobre planificación familiar durante su estadía en el hospital. La diferencia podría ser debida a una asistencia diferencial (como función de la motivación) o un recuerdo diferencial. La proyección de diapositivas y películas y charlas grabadas en cinta magnetofónica fueron vistas y oídas únicamente por una pequeña minoría de las mujeres (menos del 10\% en cada caso). Al preguntárseles cuál de las modalidades había influenciado al máximo su decisión respecto a la planificación familiar, el $38 \%$ de las aceptantes y el $43 \%$ de las no aceptantes señalaron la charla personal, (prueba de $X^{2}, p<.001$ ) mientras 19 y $10 \%$ respectivamente, mencionaron la charla de grupo (no es una diferencia estadísticamente significante).

\section{b) Reacción al mensaje:}

TABLA № 3

LO MAS IMPORTANTE APRENDIDO ACERCA DE LA PLANIFICACION FAMILIAR DURANTE LA ESTADIA EN EL HOSPITAL

\begin{tabular}{lcc}
\hline & $\begin{array}{c}\text { Aceptantes } \\
(\mathbf{n}=\mathbf{2 1 8}) \\
\text { Porcentaje }\end{array}$ & $\begin{array}{c}\text { No Aceptantes } \\
\text { (n = 193) } \\
\text { Porcentaje }\end{array}$ \\
\hline $\begin{array}{l}\text { Ventajas Económicas. } \\
\text { Espaciamiento y limitación }\end{array}$ & 26 & 15 \\
de nacimientos, salud materna. & 21 & 12 \\
No aprendió nada, o nada útil. & 15 & 21 \\
Un método específico & 11 & 1 \\
Todo fué importante. & 8 & 4 \\
Los métodos en general. & 7 & 25 \\
No recibió información o no asistió & 5 & 9 \\
a charlas de grupo. & 5 & 2 \\
No recuerda o no sabe. & 2 & 100 \\
Otros. & 100 & 9 \\
$\quad$ TOTAL & & 11 \\
\hline
\end{tabular}

Como puede verse en la Tabla $N$ : 3 , más aceptantes que no aceptantes parecen haber obtenido información de utilidad para ellas en los varios mensajes de planificación familiar. Mientras que el $73 \%$ de las aceptantes dijo que lo más importante que habían aprendido se relacionaba con un método o métodos de planificación familiar o con algún beneficio de la planificación familiar, únicamente el $57 \%$ de las no aceptantes expresaron opiniones similares (prueba de $\left.X^{2}, p<.001\right)$. El $40 \%$ de las no aceptantes vs. $25 \%$ de aceptarites dijeron no haber aprendido nada o nada útil, no haber recibido información o asistido a las charlas, o no pudieron recordar qué fué lo más importante que habían aprendido (prueba de $X^{2}, p<.01$ ).

Es interesante preguntarse si las aceptantes empezaron con una ma- 
yor convicción con respecto a las ventajas económicas y de salud de la planificación familiar y recibieron refuerzo, o si más bien fueron "convertidas" en mayor o menor grado. El conocimiento previo de tales ventajas parece más plausible y es consistente con los resultados de otros estudios. El hecho de que cuatro veces más no aceptantes citaron métodos en general, indica también que éstas han podido comenzar con me- nos información. Varios resultados parecen reflejar una mayor motivación de parte de las aceptantes; sirve de ejemplo el caso de aquellas que mencionaron un método específico, presumiblemente aquel que adoptaron más tarde.

La Tabla № 4, muestra qué información causó inquietudes a las mujeres.

TABLA NN: 4

INFORMACION SOBRE PLANIFICACION FAMILIAR QUE SORPRENDIO, CONTRARIO O PUSO A LAS MUJERES NERVIOSAS

\begin{tabular}{|c|c|c|}
\hline Información & $\begin{array}{c}\text { Aceptantes } \\
(n=196) \\
\text { Porcentaje }\end{array}$ & $\begin{array}{c}\text { No Aceptantes } \\
(n=171) \\
\text { Porcentaje }\end{array}$ \\
\hline $\begin{array}{l}\text { Nada } \\
\text { Efectos nocivos de los Orales } \\
\text { Efectos nocivos del DIU } \\
\text { Acerca de los métodos de } \\
\text { Planificación en general } \\
\text { Efectos nocivos de un método diferente } \\
\text { a los orales y el DIU } \\
\text { La necesidad de examen pélvico } \\
\text { Otra }\end{array}$ & $\begin{array}{l}37 \\
31 \\
26\end{array}$ & $\begin{array}{l}45 \\
25 \\
20\end{array}$ \\
\hline TOTAL & 100 & 100 \\
\hline
\end{tabular}

En general, las aceptantes estuvieron más preocupadas que las no aceptantes respecto a posibles complicaciones de los anticonceptivos (62 vs. $50 \%$ prueba de $\left.X^{2}: p<.001\right)$. Lo anterior presumiblemente refleja el hecho de que una no aceptante tiene menos que temer ipso facto. (Debe mencionarse que los anticonceptivos orales fueron escogidos por más del $70 \%$ de las mujeres en cada uno de los tres hospitales en la primera mitad de 1972). Los datos suministran firme apoyo a la afirmación de que el examen pélvico no actúa como un mayor obstáculo en la aceptación de planificación familiar entre esta población en particular.

\section{c) Oportunidad de hacer preguntas y de escoger libremente un método:}

A todas las mujeres se les preguntó si habían tenido oportunidad de hacer preguntas relacionadas con la planificación familiar. El $33 \%$ de las aceptantes vs. el $22 \%$ de las no aceptantes contestaron afirmativamente (prueba $X^{2}, p<.001$ ); 24 y $38 \%$ respectivamente dijeron que nó; y el 43 y $40 \%$ dijeron no haber tenido preguntas. Nuevamente, las mujeres que no asistieron a las charlas de grupo claramente habrían tenido menos oportunidad de hacer preguntas y esto puede en parte explicar la diferencia. Sinembargo, lo anterior nue- 
vamente toca la cuestión de la exposición diferencial a información sobre planificación familiar. Si las no aceptantes hubieran recibido más información o hubieran tenido más oportunidad de hacer preguntas, habría aceptado un mayor número de ellas un método de planificación familiar?

Las aceptantes que formularon preguntas estuvieron más que todo interesadas en cómo usar los métodos $(24 \%)$ mientras que el $16 \%$ de las no aceptantes preguntaron acerca de esto (prueba $X^{2}, p<.001$ ). La pregunta más común de las no aceptantes se relacionó con posibles efectos nocivos de los anticonceptivos orales $(35 \%)$; el $20 \%$ de las aceptantes también hizo esta pregunta. La diferencia tiene significancia estadística (prueba $X^{2}, p<.001$ ). El 18\% de las aceptantes y el $17 \%$ de las no aceptantes pidieron más información con el fin de escoger un método. Finalmente, el $15 \%$ de las aceptantes solicitaron consejo respecto a un método inofensivo. En un todo, los dos grupos tendieron igualmente a formular preguntas indicativas de preocupación respecto a posibles efectos nocivos ( $44 \%$ en cada grupo), aunque más no-aceptantes parecieron preocuparse acerca de los anticonceptivos orales, el método de escogencia local.

La mayoría de aceptantes ( $88 \%$ ) y no aceptantes $(86 \%)$ sintieron que habían tenido libre escogencia para adoptar un método cuando oyeron por primera vez acerca de la planificación familiar en el hospital. Sinembargo, mientras que el $74 \%$ de las aceptantes informaron que habían tenido la opción de tres o más métodos, únicamente $65 \%$ de las no aceptantes mencionaron un número igual (prueba $X^{2}, p<.001$ ). Lo anterior nuevamente hace preguntarse si la diferencia refleja motivación o exposición diferenciales o ambas cosas.

Más aceptantes (84\%) que no aceptantes (73\%) dijeron que el personal del hospital hizo un grande 0 moderado esfuerzo para convencerlas que deberían aceptar la planificación familiar (prueba $X^{2}, p<.001$ ). Esto puede reflejar un mayor esfuerzo del personal con mujeres percibidas como estando interesadas. El $4 \%$ de las aceptantes y el $5 \%$ de las no aceptantes informaron que alguien del personal del hospital les había aconsejado no aceptar la planificación familiar, citando generalmente la falta de su efectividad o efectos secundarios de los métodos.

\section{FACTORES DE CONVENIENCIA}

\section{Información respecto a Costos y Horario de la Clínica:}

El $59 \%$ de las aceptantes y el $45 \%$ de las no aceptantes dijeron haber recibido información con respecto al costo de los servicios de planificación familiar. Más aceptantes (66\%) que no aceptantes ( $50 \%$ ) informaron que habian sido notificadas acerca del horario de la Clínica. Sinembargo, una gran proporción de ambos grupos dijeron que habían sido citadas para regresar a la clínica de post-parto ( $94 \%$ de aceptantes y $83 \%$ de no aceptantes).

\section{Información respecto a la estadía en el Hospital:}

Se ha planteado frecuentemente la hipótesis de que la aceptación de planificación familiar está positivamente asociada con el término de estadía en el hospital. Lo anterior no fue corroborado por nuestros resultados: $61 \%$ de las no aceptantes vs. $55 \%$ de las aceptantes estuvieron por más de 48 horas. Sinembargo, un poco más de no aceptantes $(54 \%)$ que de acep- 
tantes ( $49 \%$ ) estuvieron en el hospital durante un fin de semana.

\section{Conveniencia de la Clínica de Post-Parto:}

No hubo diferencias significativas en las mediciones objetivas de la conveniencia de la Clínica: $69 \%$ de aceptantes y $66 \%$ de no aceptantes podían llegar a la Clínica en menos de media hora; el 75 y $78 \%$ respectivamente viajaban en bus; el 67 y el $79 \%$ respectivamente tenían que pagar menos de tres pesos para transportarse. Sinembargo, mientras que el $30 \%$ de las no aceptantes reportaron tener dificultad en conseguir una niñera, el $46 \%$ de las aceptantes tenían este problema.

\section{Satisfacción de las Aceptantes con los Servicios de la Clínica:}

En general, las aceptantes parecieron estar satisfechas con el servicio en las Clínicas. El $98 \%$ calificaron los servicios como "buenos", aunque las únicas otras respuestas posibles eran "pobre" y "no estoy segura". El $84 \%$ dijeron haber tenido tiempo suficiente para discutir el método así como sus problemas de planificación familiar con el personal clínico. El $73 \%$ afirmaron que no les había molestado el tiempo de espera, 95\% dijeron haber tenido suficiente intimidad durante el examen y el $87 \%$ lo mismo durante sus conversaciones con el personal clínico. Al preguntárseles acerca del costo de los servicios, $74 \%$ consideraron el precio justo y el $24 \%$ muy bajo. En el momento de la entrevista, el $88 \%$ todavía estaba usando el método aceptado. Finalmente, $60 \%$ de las aceptantes habían recomendado los servicios de planificación familiar a sus amigas o parientes.

\section{Discusión}

En nuestro informe sobre la relación entre características socio-demográficas y aceptación (1), la fuerte influencia de la paridad y las opiniones del esposo y la madre fueron obvias: una mayor paridad y opiniones favorables estaban positivamente asociadas con una mayor aceptación. Resultados positivos similares no emergen de los datos presentados aquí, lo cual puede ser en parte una función de la "suavidad" de las variables y de la variedad de relaciones causales positivas. Claramente, el conocimiento sobre planificación familiar y percepciones sobre sus ventajas o el conocimiento de la conveniencia de la Clínica, no se prestan fácilmente a que se mida su efecto sobre la aceptación. Sinembargo, sí emergieron algunos resultados bien definidos: la duración de estadía en el hospital no estuvo positivamente relacionada con la aceptación; varios factores de conveniencia de la clínica (vgr. tiempo de viaje y costo) no se diferenciaron entre aceptantes y no aceptantes; y en esta población, el examen pélvico no pareció actuar como obstáculo de la aceptación.

El estudio apoyó la hipótesis de que el conocimiento de la planificación familiar está positivamente asociado con la aceptación. En general, las aceptantes habían sabido acerca de la disponibilidad de servicios de planificación familiar por un tiempo más largo, tuvieron mayores probabilidades de haberse enterado de los servicios por intermedio de amigas y parientes y de conocer a una o más de éstas quienes estaban usándolos. Lo anterior proporciona evidencia adicional sobre la influencia de amigas y parientes sobre la aceptación. Cada uno de estos resultados encaja dentro de las diferentes etapas de adopción de una innovación postuladas en el 
modelo de difusión de Rogers (4). Las no aceptantes en promedio poseían menor conocimiento y quizás no habían avanzado suficientemente en el proceso de adopción como para adoptar un método. El apoyo para esta noción se deriva del hecho de que el $35 \%$ indicaron intenciones futuras de practicar la planificación familiar y otro $46 \%$ dijeron que no sabían; únicamente un $13 \%$ aseguraron no tener tales planes. Fue también interesante notar que el $25 \%$ de no aceptantes vs. $7 \%$ de aceptantes citaron métodos de planificación familiar en general como lo más importante que habían aprendido en el hospital acerca de la planificación familiar, lo cual ciertamente es indicativo de interés.

Es difícil interpretar los resultados relacionados con la reacción al mensaje de planificación familiar. Las aceptantes en general estuvieron más propensas a identificar algo positivo como lo más importante que habían aprendido. Sinembargo, si lo anterior se debió a mayores conocimientos y motivación previos respecto a la planificación familiar, es una pregunta debatible que requiere investigación adicional. Tampoco podemos decir mucho respecto a los diferentes medios de comunicación, ya que únicamente se emplearon charlas personales y de grupo. Sinembargo, hubo alguna indicación de que las charlas personales fueron más efectivas en esta población particular.

Hubo algunas indicaciones de que las no aceptantes abrigaban mayores preocupaciones relacionadas con posibles efectos nocivos de la anticoncepción y pudieron haber estado restringidas en la aceptación debido en parte a esta razón. Por ejemplo, de las mujeres que hicieron preguntas, el $35 \%$ de no aceptantes vs. $20 \%$ de aceptantes preguntaron acerca de po- sibles efectos nocivos de los anticonceptivos orales. En su mayoría las aceptantes parecieron satisfechas con los servicios de planificación farniliar, haciendo poco probable el que la reputación de la clínica obstaculizara la aceptación.

El estudio en un todo indicó que las opiniones favorables del esposo y la madre, mayor paridad y mayor conocimiento de la planificación familiar fueron los factores más importantes que influyeron en la aceptación.

\section{Conclusiones}

Cierto número de conclusiones prácticas importantes se desprenden de los resultados presentados anteriormente:

1. Los hospitales que ofrecen atención de maternidad podrían provechosamente emprender experimentos en pequeña escala con el fin de identificar los medios más efectivos de suministrar información y educación en planificación familiar a mujeres en el post-parto y sus esposos.

2. El mensaje de planificación familiar tiene tendencia a ser más efectivo si está orientado hacia los beneficios económicos y de salud de la anticoncepción, provee información detallada respecto a los métodos y sus posibles complicaciones e información sobre cuándo, dónde y a qué costo están disponibles los servicios.

3. La definición de "no aceptantes" debe entenderse en términos relativos ya que parece claro que muchas mujeres en estas categorías se encuentran en distintas etapas en camino hacia la aceptación. Parece lógico por lo tanto, recomendar que éstas sean seguidas, que les sea ofrecicla información adicional y que se les 
ofrezcan los servicios a intervalos regulares.

4. Dada la influencia sobre la aceptación, de amigas y parientes, especialmente de aquellas que están usando los servicios de planificación familiar, los administradores podrían considerar involucrar aceptantes en los programas de información y educación, sea como motivadoras en la sala de maternidad o como trabajadoras en la comunidad.

\section{Conclusions}

A number of important practical conclusions follow from the findings presented:

1. Hospitals which offer maternity care could profitably undertake small scale experiments to identify the most effective means of providing family planning information and education to postpartum women and their spouses.

2. The family planning message is likely to be more effective if oriented toward the health and economic benefits of contraception, provides detailed information regarding methods and their possible side effects, and information regarding where, when and at what cost services are available.

3. The definition of "non-acceptor" should be understood in relative terms since it seems clear that many women in this category are at various stages en route to acceptance. It seems logical, therefore, to recommend that they be followed-up, provided with further information and offered services at regular intervals.

4. Given the influence on acceptance of friends and relatives, especially those using the family planning services, administrators may with to consider involving acceptors in the information and education programs, either as ward motivators or community workers.

\section{BIBLIOGRAFIA}

I MARTINEZ E. Y MEASHAN, A. Revista Colombiana de Obstetricia y Ginecología. Vol. 14, No 3: 185-194 (1973).

2 Esta y otras dificultades metodológicas se discuten en la Referencia 1.

3 ROSS, J. y otros. Informes sobre PoblaciónPlanificación Familiar. 12: 17, 1972.

4 ROGERS, E. M., Diffusion of Innovation, (Glencoe, N.Y., Free Press of Glencoe, 1962). 